Discussion

Papers

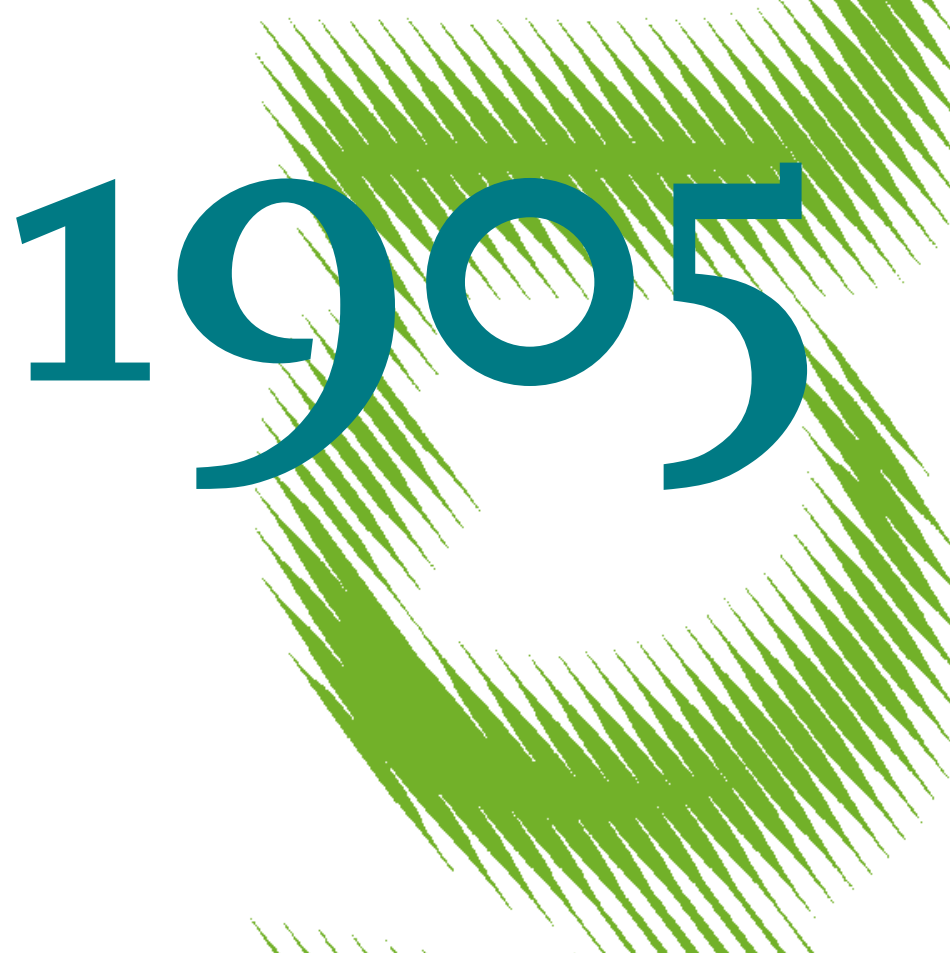

A Simple Instrument for Proxy

Vector Autoregressive Analysis 
Opinions expressed in this paper are those of the author(s) and do not necessarily reflect views of the institute.

IMPRESSUM

(C) DIW Berlin, 2020

DIW Berlin

German Institute for Economic Research

Mohrenstr. 58

10117 Berlin

Tel. +49 (30) $89789-0$

Fax +49 (30) $89789-200$

http://www.diw.de

ISSN electronic edition 1619-4535

Papers can be downloaded free of charge from the DIW Berlin website:

http://www.diw.de/discussionpapers

Discussion Papers of DIW Berlin are indexed in RePEc and SSRN:

http://ideas.repec.org/s/diw/diwwpp.html

http://www.ssrn.com/link/DIW-Berlin-German-Inst-Econ-Res.html 


\title{
A Simple Instrument for Proxy Vector Autoregressive Analysis
}

\author{
by \\ Lukas Boer ${ }^{1}$ \\ DIW Berlin and Humboldt-Univeristät Berlin, Mohrenstr. 58, 10117 Berlin, \\ Germany \\ email: lboer@diw.de \\ and \\ Helmut Lütkepohl \\ DIW Berlin and Freie Universität Berlin, Mohrenstr. 58, 10117 Berlin, \\ Germany \\ email: hluetkepohl@diw.de
}

October 5, 2020

\begin{abstract}
A major challenge for proxy vector autoregressive analysis is the construction of a suitable instrument variable for identifying a shock of interest. We propose a simple proxy that can be constructed whenever the dating and sign of particular shocks are known. It is shown that the proxy can lead to impulse response estimates of the impact effects of the shock of interest that are nearly as efficient as or even more efficient than estimators based on a conventional, more sophisticated proxy.
\end{abstract}

Key Words: GMM, heteroskedastic VAR, instrumental variable estimation, proxy VAR, structural vector autoregression.

JEL classification: C32

\footnotetext{
${ }^{1}$ We thank Jonathan Wright for making the Matlab code for his analysis available and Malte Rieth for comments on an earlier draft of the paper.
} 


\section{Introduction}

In proxy vector autoregressive (VAR) analysis, an instrument variable is used to identify the impact effects of a structural shock of interest. The instrument or proxy has to be relevant for the shock of interest and it has to be exogenous to all other structural shocks. In other words, the proxy has to be correlated with the shock of interest and it has to be uncorrelated with all other structural shocks. Although proxy VAR analysis has become quite popular lately (e.g., Stock and Watson (2012), Mertens and Ravn (2013), Gertler and Karadi (2015), Piffer and Podstawski (2018), Caldara and Herbst (2019), and further references in Kilian and Lütkepohl (2017, Chapter 15)), the construction of a suitable proxy is often a main challenge because it requires additional information from other sources than those captured in the model.

As an alternative to constructing a proxy, one may consider using an approach that was suggested by Wright (2012). He proposes to identify a structural shock from the change in its variance due to specific events. For example, a monetary policy shock may be more volatile at dates of central bank council meetings. Using the heteroskedasticity for identification, only the dates of the specific events must be known and there is no need to construct a proper instrument variable associated with the specific events.

In this study, we propose to construct a dummy variable that assumes values \pm 1 if a positive or negative shock occurs at the dates of the special events, respectively, and that is zero otherwise. Hence, all we need to construct this dummy variable is knowledge of the dates of the special events, like in Wright's approach, and the signs of the possible shocks that may have occurred at the event dates. Such knowledge is available for a number of shocks that have been used in structural VAR analysis. For example, some crises in the Middle East are known to have caused disruptions in oil supply. Such information can be employed to construct a dummy variable for identifying oil supply shocks. Likewise, there are a number of events such as the 9/11 attacks on the US that have caused increases in economic uncertainty and could be used for constructing a sign-based dummy to identify uncertainty shocks.

Through simulation, we show that such dummy variables, referred to as sign-proxies in the following, can be used as proxies for identifying structural shocks and that they may yield estimates of the impact effects of the structural shocks of interest that are more precise in terms of root mean squared error (RMSE) than the estimates obtained by Wright's approach. In fact, the sign-proxies often provide more precise estimates than conventional, more sophisticated proxies that are not strongly correlated with the shock of in- 
terest. We use a model from Wright (2012) to illustrate the benefits in terms of estimation efficiency from using a sign-proxy.

In Wright's approach and in the standard proxy VAR approach, the impact effects of the shock of interest and, hence, its impulse responses are typically estimated by the generalized method of moments (GMM). Because using more moment conditions may lead to more efficient GMM estimators and since the two approaches for estimating the impact effects of the shock of interest use different sets of moment conditions, combining the moment conditions may lead to efficiency gains. Therefore we also consider this combination approach. It turns out, however, that in the present situation, the combination approach does not lead to uniform improvements of estimation efficiency for the impulse responses.

The remainder of the paper is structured as follows. In the next section, the general model setup is presented and the different estimators of the impulse responses are discussed. In Section 3, a Monte Carlo experiment is conducted to compare the small sample performance of the estimators. In Section 4, a model due to Wright (2012) is reconsidered and the advantage of using a sign-proxy is demonstrated. Conclusions are presented in the final section.

\section{The Structural VAR Setup}

A $K$-dimensional reduced-form VAR model

$$
y_{t}=\nu+A_{1} y_{t-1}+\cdots+A_{p} y_{t-p}+u_{t}
$$

is considered, where $u_{t}$ is a zero mean white noise process with two volatility regimes associated with covariance matrices $\Sigma_{0}$ and $\Sigma_{1}$. The matrix $\Sigma_{0}$ is the usual covariance and it changes to $\Sigma_{1}$ in $M$ periods, where specific events occur or announcements are made, i.e., when $t \in \mathcal{T}_{1}=\left\{t_{a_{1}}, \ldots, t_{a_{M}}\right\}$. In other words, $\mathbb{E}\left(u_{t} u_{t}^{\prime}\right)=\Sigma_{0}$ for $t \notin \mathcal{T}_{1}$ and $\mathbb{E}\left(u_{t} u_{t}^{\prime}\right)=\Sigma_{1}$ for $t \in \mathcal{T}_{1}$. A sample $y_{t}, t \in \mathcal{T}=\{1, \ldots, T\}$, is assumed to be available for inference.

The structural errors, $w_{t}=\left(w_{1 t}, \ldots, w_{K t}\right)^{\prime}$, are obtained from the reducedform errors by a linear transformation $w_{t}=B^{-1} u_{t}$ or $u_{t}=B w_{t}$ such that the structural matrix $B$ represents the impact effects of the structural shocks on the variables $y_{t}$. The structural errors are assumed to be instantaneously uncorrelated, i.e., $w_{t}$ has a diagonal covariance matrix.

Without loss of generality it is assumed that the first structural shock, $w_{1 t}$, is the shock of specific interest and it is normalized such that it has a unit impact effect on the first variable, whereas the other shocks, $w_{2 t}, \ldots, w_{K t}$, 
have unit variances. In other words, $w_{t}$ is a white noise process with zero mean and covariance matrix

$$
\Sigma_{0}^{w}=\left[\begin{array}{cc}
\sigma_{0}^{2} & 0 \\
0 & I_{K-1}
\end{array}\right] \text { for } t \in \mathcal{T} \backslash \mathcal{T}_{1} \quad \text { and } \quad \Sigma_{1}^{w}=\left[\begin{array}{cc}
\sigma_{1}^{2} & 0 \\
0 & I_{K-1}
\end{array}\right] \text { for } t \in \mathcal{T}_{1}
$$

Note that this setup, where only the variance of the first structural shock changes, is not very common in the structural VAR literature. It is, however, the basis for Wright's heteroskedasticity approach to work, as explained later. In contrast, the proxy VAR approach works under more general assumptions. We still make the more restrictive assumption here to compare the proxy VAR approach to Wright's heteroskedasticity approach.

Denoting by $\mathbf{b}_{i}$ the $i^{\text {th }}$ column of $B$, i.e., $B=\left[\mathbf{b}_{1}, \ldots, \mathbf{b}_{K}\right]$, the relation $u_{t}=B w_{t}=\sum_{k=1}^{K} \mathbf{b}_{k} w_{k t}$ implies under the present assumptions that

$$
\Sigma_{0}=\sigma_{0}^{2} \mathbf{b}_{1} \mathbf{b}_{1}^{\prime}+\sum_{k=2}^{K} \mathbf{b}_{k} \mathbf{b}_{k}^{\prime} \quad \text { and } \quad \Sigma_{1}=\sigma_{1}^{2} \mathbf{b}_{1} \mathbf{b}_{1}^{\prime}+\sum_{k=2}^{K} \mathbf{b}_{k} \mathbf{b}_{k}^{\prime} .
$$

Hence,

$$
\Sigma_{1}-\Sigma_{0}=c_{W} \mathbf{b}_{1} \mathbf{b}_{1}^{\prime},
$$

where $c_{W}=\sigma_{1}^{2}-\sigma_{0}^{2}$. The vector $\mathbf{b}_{1}$ contains the structural parameters of interest. If $\mathbf{b}_{1}$ and the reduced-form VAR parameters from equation (1) are known, the responses of the variables $y_{t}$ to the first structural shock can be traced over time using the relations

$$
\theta_{h}=\Phi_{h} \mathbf{b}_{1}, \quad h=1,2, \ldots,
$$

where $\theta_{h}$ is a $(K \times 1)$ vector of structural impulse responses for propagation horizon $h$ and $\Phi_{i}=\sum_{j=1}^{i} \Phi_{i-j} A_{j}$ can be obtained recursively for $h=0,1, \ldots$, from the VAR slope coefficients starting with $\Phi_{0}=I_{K}$ (Lütkepohl, 2005, Section 2.1.2). Because estimation of the reduced-form VAR model in expression (1) is straightforward, the focus of interest in the following is precise estimation of the structural parameters $\mathbf{b}_{1}$.

As mentioned earlier, the first element of $\mathbf{b}_{1}$ is standardized to equal 1 . In other words, the impact effect of the first structural shock on the first variable is standardized to one. This assumption is just a normalization and does not entail a loss of generality if the first element of $\mathbf{b}_{1}$ is nonzero. Denoting the last $K-1$ elements of $\mathbf{b}_{1}$ by $\mathbf{b}_{12}$ such that $\mathbf{b}_{1}^{\prime}=\left(1, \mathbf{b}_{12}^{\prime}\right)$, we focus on estimators of $\mathbf{b}_{12}$ in the following. 


\subsection{The Proxy VAR Approach}

The fact that the volatility of the first structural shock changes for periods $t \in \mathcal{T}_{1}$ suggests that special shocks $w_{1 t}$ occur in event periods. Based on this observation, it may be possible to find an instrument that can be used for estimating $\mathbf{b}_{12}$.

Suppose there is an instrumental (proxy) variable $z_{t}$ satisfying

$$
\begin{aligned}
& \mathbb{E}\left(w_{1 t} z_{t}\right)=c \neq 0 \quad \text { (relevance), } \\
& \mathbb{E}\left(w_{k t} z_{t}\right)=0, \quad k=2, \ldots, K \quad \text { (exogeneity). }
\end{aligned}
$$

In that case, a multiple of $\mathbf{b}_{1}$ can be estimated by using $z_{t}$ as an instrument and observing that $\mathbb{E}\left(u_{t} z_{t}\right)=c \mathbf{b}_{1}$. This setup assumes, however, that the covariance $\mathbb{E}\left(u_{t} z_{t}\right)$ is not affected by the change in the volatility of $u_{t}$. In that case, the relations in (4) and (5) provide moment conditions for estimating a multiple of $\mathbf{b}_{1}$. An estimator for $\mathbf{b}_{1}$ may then be obtained by dividing all elements of the estimator of $c \mathbf{b}_{1}$ by the first element.

More precisely, a GMM estimator is obtained by estimating the reducedform VAR in expression (1) by equation-wise ordinary least squares (OLS) and using the proxy VAR estimator

$$
\widehat{\mathbf{b}}_{12}^{P}=\left(\frac{\sum_{t=1}^{T} \hat{u}_{2 t} z_{t}}{\sum_{t=1}^{T} \hat{u}_{1 t} z_{t}}, \ldots, \frac{\sum_{t=1}^{T} \hat{u}_{K t} z_{t}}{\sum_{t=1}^{T} \hat{u}_{1 t} z_{t}}\right)^{\prime}
$$

for $\mathbf{b}_{12}$. Here $\hat{u}_{t}=\left(\hat{u}_{1 t}, \ldots, \hat{u}_{K t}\right)^{\prime}$ are the OLS residuals.

The instrument may be suggested by the subject matter. For example, Piffer and Podstawski (2018) use changes in the price of gold to construct an instrument for uncertainty shocks and Cesa-Bianchi, Thwaites and Vicondoa (2020) construct a time series of intra-day price variation of the 3-month Sterling future contracts around policy decisions of the Monetary Policy Committee of the Bank of England as a proxy for monetary policy shocks. However, finding a good proxy that satisfies conditions (4) and (5) for a shock of interest is not always easy. This problem is reflected in the fact that, in some studies, the instrument is only available for a shorter period than the sample period of the other variables in the model (see, e.g., Gertler and Karadi (2015)).

If only certain dates of announcements or special events are available, where specific shocks were transmitted, one may construct a simple dummy variable

$$
s_{t}=\left\{\begin{array}{cl}
\operatorname{sgn}\left(w_{1 t}\right) \cdot 1 & \text { for } \quad t \in \mathcal{T}_{1}, \\
0 & \text { for } \quad t \notin \mathcal{T}_{1},
\end{array}\right.
$$


where $\operatorname{sgn}(\cdot)$ denotes the sign function which assigns the sign of its argument. Thus, $s_{t}$ assumes a value of +1 or -1 depending on whether the special event induces a positive or negative shock. In periods without known special events, $s_{t}=0$. Note that, if the shocks $w_{1 t}, \ldots, w_{K t}$ are stochastically independent, e.g., if they are Gaussian as sometimes assumed in the literature, $s_{t}$ will also be independent of $w_{2 t}, \ldots, w_{K t}$ (see, e.g., Mood, Graybill and Boes (1974, p. 151, Theorem 3)). Hence, $s_{t}$ qualifies as a proxy.

Constructing the sign-proxy $s_{t}$ in this way assumes that the investigator at least knows whether a positive or negative shock was induced in a specific period. It may actually not always be clear which shocks are positive and which ones are negative because the sign of a shock is typically linked to some economic variable. For example, a positive (expansionary) monetary policy shock is often associated with a reduction in the policy interest rate. However, this indicator for monetary policy shocks is not available in times of zero interest rates. For those periods, some other indicator is needed to determine whether a policy shock is positive or negative. For example, expansions in bond purchases may be linked to expansionary monetary policy shocks. Of course, the sign of the shock may be inferred from a set of variables. The important precondition for constructing the sign-proxy $s_{t}$ is that the researcher knows the date and the sign of the structural shock on specific event dates.

It is also important to note that the signs of some proxy $z_{t}$ and a signproxy $s_{t}$ for the same shock may differ. As both proxies are correlated with the first structural shock, $w_{1 t}, z_{t}$ and $s_{t}$ will also be correlated with each other. This does not mean, however, that they always assume values of the same sign. It may be worth noting that constructing the dummy with reverse signs makes no difference for our purposes. In other words, we may define

$$
s_{t}=\left\{\begin{array}{cl}
-\operatorname{sgn}\left(w_{1 t}\right) \cdot 1 & \text { for } t \in \mathcal{T}_{1}, \\
0 & \text { for } t \notin \mathcal{T}_{1},
\end{array}\right.
$$

instead of using the $s_{t}$ defined in expression (7). The crucial property of $s_{t}$ is that it has to be correlated with $w_{1 t}$. Whether or not the correlation is positive or negative is not important for its usefulness as an instrument.

In fact, we also consider the situation where a researcher does not know the sign of the shock for some event dates for sure and, hence, may occasionally assign signs incorrectly. We denote by $s_{t}^{(m)}$ a sign-proxy for which $m$ signs are classified incorrectly. Clearly, misspecifying some signs may undermine the correlation between the shock of interest and the sign-proxy and, hence, it may weaken the proxy as an instrument for the shock.

The sign-proxy in (7) is clearly related to the specific shocks induced by the special events and, thus, can be used just like a regular proxy to estimate 
the impact effects of the first shock $w_{1 t}$. In other words, the associated sign-proxy estimator is

$$
\widehat{\mathbf{b}}_{12}^{S P}=\left(\frac{\sum_{t=1}^{T} \hat{u}_{2 t} s_{t}}{\sum_{t=1}^{T} \hat{u}_{1 t} s_{t}}, \ldots, \frac{\sum_{t=1}^{T} \hat{u}_{K t} s_{t}}{\sum_{t=1}^{T} \hat{u}_{1 t} s_{t}}\right)^{\prime} .
$$

If $s_{t}$ is replaced by $s_{t}^{(m)}$, the corresponding estimator is denoted by $\widehat{\mathbf{b}}_{12}^{S P(m)}$.

\subsection{Wright's Heteroskedasticity Approach}

Wright (2012) proposes another approach to estimate the impact effects of the shock of interest. It has the advantage that just the dates of special events or announcements have to be known. He suggests using the moment conditions related to the variance change in (3) for GMM estimation. More precisely, he proposes to minimize a GMM objective function analogous to

$$
\begin{aligned}
J_{W}\left(\mathbf{b}_{12}, c_{W}\right)= & \operatorname{vech}\left(\widehat{\Sigma}_{1}-\widehat{\Sigma}_{0}-c_{W} \mathbf{b}_{1} \mathbf{b}_{1}^{\prime}\right)^{\prime}\left(\frac{\widehat{\Omega}_{0}}{T-M}+\frac{\widehat{\Omega}_{1}}{M}\right)^{-1} \\
& \times \operatorname{vech}\left(\widehat{\Sigma}_{1}-\widehat{\Sigma}_{0}-c_{W} \mathbf{b}_{1} \mathbf{b}_{1}^{\prime}\right)
\end{aligned}
$$

with respect to the last $K-1$ elements of $\mathbf{b}_{1}$ and $c_{W}$. Here

$$
\widehat{\Omega}_{0}=\frac{1}{T-M} \sum_{t \in \mathcal{T} \backslash \mathcal{T}_{1}} \operatorname{vech}\left(\hat{u}_{t} \hat{u}_{t}^{\prime}-\overline{\hat{u} \hat{u}^{\prime}}\right) \operatorname{vech}\left(\hat{u}_{t} \hat{u}_{t}^{\prime}-\overline{\hat{u} \hat{u}^{\prime}}\right)^{\prime}
$$

and

$$
\widehat{\Omega}_{1}=\frac{1}{M} \sum_{t \in \mathcal{T}_{1}} \operatorname{vech}\left(\hat{u}_{t} \hat{u}_{t}^{\prime}-\overline{\hat{u}} \hat{u}^{\prime}\right) \operatorname{vech}\left(\hat{u}_{t} \hat{u}_{t}^{\prime}-\overline{\hat{u} \hat{u}^{\prime}}\right)^{\prime} .
$$

The resulting estimator of $\mathbf{b}_{12}$ is denoted by $\widehat{\mathbf{b}}_{12}^{W}$.

It is important to note that, although the moment conditions in equation (3) and in (4)/(5) both specify a multiple of $\mathbf{b}_{1}$, they may not specify the same multiple of $\mathbf{b}_{1}$. In other words, $c$ and $c_{W}$ may be distinct.

\subsection{Joint GMM}

Because more moment conditions may improve the efficiency of GMM estimators, it may make sense to consider the joint moment conditions

$$
m\left(\mathbf{b}_{12}, c_{W}, c\right)=\left[\begin{array}{c}
\mathbb{E}\left(u_{t} z_{t}\right)-c \mathbf{b}_{1} \\
\operatorname{vech}\left(\Sigma_{1}-\Sigma_{0}-c_{W} \mathbf{b}_{1} \mathbf{b}_{1}^{\prime}\right)
\end{array}\right]=0
$$


or to consider these moments relying on the sign-proxy and, hence, replacing $\mathbb{E}\left(u_{t} z_{t}\right)$ in the first block of moments by $\mathbb{E}\left(u_{t} s_{t}\right)$. Using

$$
\overline{\hat{u} z}=\frac{1}{T} \sum_{t=1}^{T} \hat{u}_{t} z_{t} \quad \text { and } \quad \overline{\hat{u} s}=\frac{1}{T} \sum_{t=1}^{T} \hat{u}_{t} s_{t}
$$

as estimators of $\mathbb{E}\left(u_{t} z_{t}\right)$ and $\mathbb{E}\left(u_{t} s_{t}\right)$, respectively, the GMM estimator is obtained by minimizing the objective function

$$
J_{g e n}\left(\mathbf{b}_{12}, c_{W}, c\right)=\widehat{m}\left(\mathbf{b}_{12}, c_{W}, c\right)^{\prime} \widehat{\Omega}_{m}^{-1} \widehat{m}\left(\mathbf{b}_{12}, c_{W}, c\right),
$$

where $\widehat{\Omega}_{m}$ is an estimator of the covariance matrix of the estimated moment conditions $\widehat{m}\left(\mathbf{b}_{12}, c_{W}, c\right)$. For example, for the proxy $z_{t}$, assuming that the first and second part of the estimated moment conditions are asymptotically independent, suggests a GMM objective function

$$
J\left(\mathbf{b}_{12}, c_{W}, c\right)=T\left(\overline{\hat{u} z}-c \mathbf{b}_{1}\right)^{\prime} \widehat{\Omega}_{u z}^{-1}\left(\overline{\hat{u} z}-c \mathbf{b}_{1}\right)+J_{W}\left(\mathbf{b}_{12}, c_{W}\right),
$$

where

$$
\widehat{\Omega}_{u z}=\frac{1}{T} \sum_{t=1}^{T}\left(\hat{u}_{t} z_{t}-\overline{\hat{u} z}\right)\left(\hat{u}_{t} z_{t}-\overline{\hat{u} z}\right)^{\prime} .
$$

Equivalently, one could minimize the objective function $J_{g e n}\left(\mathbf{b}_{12}, c_{W}, c\right)$ with weighting matrix

$$
\widehat{\Omega}_{m}=\left[\begin{array}{cc}
\frac{\widehat{\Omega}_{u z}}{T} & 0 \\
0 & \frac{\widehat{\Omega}_{0}}{T-M}+\frac{\widehat{\Omega}_{1}}{M}
\end{array}\right] .
$$

The resulting estimator is denoted by $\widehat{\mathbf{b}}_{12}^{W P}$. Likewise, if the proxy $z_{t}$ is replaced by the sign-proxy $s_{t}$, the corresponding estimator is denoted by $\widehat{\mathbf{b}}_{12}^{W S P}$.

In the next section, the estimators $\widehat{\mathbf{b}}_{12}^{P}, \widehat{\mathbf{b}}_{12}^{S P}, \widehat{\mathbf{b}}_{12}^{W}, \widehat{\mathbf{b}}_{12}^{W P}$, and $\widehat{\mathbf{b}}_{12}^{W S P}$ of the impact effects of the first structural shock are compared in a simulation study.

\section{Monte Carlo Investigation of Estimator Ef- ficiency}

\subsection{Monte Carlo Design}

For the Monte Carlo experiment, we have used different VAR data generating processes (DGPs), all giving qualitatively similar results. Therefore, 
we just present representative results for a DGP that is based on the empirical example of Wright (2012). He considers a 6-dimensional VAR(1) process for six different daily US interest rate variables estimated with data from November 3, 2008, through September 30, 2011 (for more details see Section 4). His sample size is $T=730$. We use his dataset and model and we use the estimated parameters for our DGP. The constant term is $\nu=(0.156,0.059,0.030,0.128,0.184,0.225)^{\prime}$ and the slope coefficient matrix is

$$
A_{1}=\left(\begin{array}{rrrrrr}
1.028 & -0.003 & 0.023 & -0.014 & 0.032 & -0.090 \\
0.040 & 0.947 & 0.011 & -0.008 & 0.022 & -0.058 \\
0.029 & -0.041 & 1.001 & -0.003 & 0.016 & -0.039 \\
0.019 & 0.000 & 0.006 & 0.947 & -0.002 & -0.008 \\
0.008 & 0.007 & 0.003 & -0.027 & 0.998 & -0.028 \\
0.046 & -0.008 & 0.017 & -0.011 & 0.031 & 0.886
\end{array}\right)
$$

Its eigenvalues have a maximum modulus of 0.994 and, hence, the DGP is stable but very persistent with several autoregressive roots very close to the unit circle. We use samples of different size generated with this VAR(1) DGP.

The impact effects matrix $B$ is constructed using the Cholesky decomposition of the estimated residual covariance matrix, $T^{-1} \sum_{t=1}^{T} \hat{u}_{t} \hat{u}_{t}^{\prime}$, and dividing the first column by the upper left-hand element such that the first column of $B$ has a first element equal to one, as assumed in Section 2.

Wright (2012, Table 5) also constructs an instrument $z_{t}$ for a monetary policy shock related to $M=28$ event days. He uses the first principal component of a set of bond futures traded at the Chicago Mercantile Exchange on the announcement days and constructs a proxy for monetary policy shocks on that basis. We construct our proxy such that it has similar properties using

$$
z_{t}=w_{1 t}+v_{t} \quad \text { for } \quad t \in \mathcal{T}_{1}
$$

where $v_{t}$ is a measurement error that is assumed to be independent of $w_{1 t}$. This type of proxy is also assumed in other proxy VAR studies (e.g., Caldara and Herbst (2019), Lütkepohl and Schlaak (2020)). Note that the correlation between the proxy and the first structural shock, $\operatorname{corr}\left(z_{t}, w_{1 t}\right)=\sigma_{1} / \sqrt{\sigma_{1}^{2}+\sigma_{v}^{2}}$ for $t \in \mathcal{T}_{1}$, depends on the variance $\sigma_{v}^{2}$ of $v_{t}$. Hence, the strength of the instrument will also depend on $\sigma_{v}^{2}$. In the simulations, we use Gaussian $v_{t}$, i.e., $v_{t} \sim \mathcal{N}\left(0, \sigma_{v}^{2}\right)$, and choose values for $\sigma_{v}^{2}$ such that the correlation between $z_{t}$ and $w_{1 t}$ is either 0.9 or 0.7 on event dates $t \in \mathcal{T}_{1}$. The former value represents high correlation and is chosen to obtain a strong instrument 
while the second value of 0.7 results in a weaker instrument, as we will see in the simulations where also tests for strong instruments are reported.

As we suspect that the performance of the different estimators depends to some extent on the difference between the variances in the two regimes $\left(\sigma_{0}^{2}\right.$ and $\left.\sigma_{1}^{2}\right)$ and the number of announcement periods, $M$, of the sample, we vary these characteristics of the first structural shock in constructing our proxy. More precisely, we construct the first shock as

$$
w_{1 t}= \begin{cases}\mathcal{N}\left(0, \sigma_{1}^{2}\right) & \text { for } \quad t \in \mathcal{T}_{1}, \\ \mathcal{N}\left(0, \sigma_{0}^{2}\right) & \text { for } \quad t \in \mathcal{T} \backslash \mathcal{T}_{1},\end{cases}
$$

where we set $\sigma_{0}^{2}=1$ and assign either the value 4 or the value 10 to $\sigma_{1}^{2}$. Thus, the standard deviations in the more volatile regimes are 2 and 3.16. In other words, on event dates the shocks are either twice or about three times as volatile as in other periods. The other shocks are also Gaussian, $\left(w_{2 t}, \ldots, w_{K t}\right) \sim \mathcal{N}\left(0, I_{K-1}\right)$. The instruments $z_{t}$ and $s_{t}$ are constructed based on these structural shocks as in (10) and (7), respectively. For $s_{t}$ we also allow for the possibility that some shocks are classified incorrectly as positive or negative. In that case we denote the estimator by $\widehat{\mathbf{b}}_{12}^{S P(m)}$, where $m$ signifies the number of incorrect sign assignments used for the sign-proxy.

The performance criteria for comparing different estimators are linked to the last $K-1$ elements of $\mathbf{b}_{1}$, i.e., to $\mathbf{b}_{12}$. We consider the root mean squared errors (RMSEs) of the estimators for these elements, $\widehat{\mathbf{b}}_{12}^{S P}, \widehat{\mathbf{b}}_{12}^{S P(m)}$, $\widehat{\mathbf{b}}_{12}^{W}, \widehat{\mathbf{b}}_{12}^{W P}$, and $\widehat{\mathbf{b}}_{12}^{W S P}$, relative to the RMSE of $\widehat{\mathbf{b}}_{12}^{P}$. To compute the relative RMSE of an estimator such as $\widehat{\mathbf{b}}_{12}^{S P}$, we divide the RMSE of each element of $\widehat{\mathbf{b}}_{12}^{S P}$ by the RMSE of the corresponding element of $\widehat{\mathbf{b}}_{12}^{P}$ and then add these relative RMSEs to get the relative RMSE of the whole estimated vector $\widehat{\mathbf{b}}_{12}^{S P}$. Thereby we control for differences in the RMSEs of the individual elements of a vector. For each simulation design, the experiment is repeated 10,000 times.

\subsection{Simulation Results}

Before looking at the relative RMSEs of the estimators, it may be worth assessing the strengths of the different instruments. This should give a first indication concerning the suitability of the different estimators for empirical exercises.

\subsubsection{Instrument Strength}

We report the relative frequencies of heteroskedasticity-robust $F$-statistics for weak instruments being smaller than 10 in our simulations (see Stock, 
Wright and Yogo (2002) or Kilian and Lütkepohl (2017, Section 15.2.1)). ${ }^{2}$ A threshold value of 10 is typically used in the related literature to classify an instrument as being sufficiently strong. Since our data are heteroskedastic, we use a robust $F$-statistic. In Table 1 the relative frequencies of test values below 10 are reported for the different proxies.

Table 1: Relative Frequencies of Heteroskedasticity-Robust Weak Instrument $F$-test Statistics Smaller than 10 (in \%)

\begin{tabular}{cccrrrrrr}
\hline \hline & & & \multicolumn{6}{c}{ Proxy variables } \\
\cline { 3 - 9 } Sample size $T$ & $M$ & $\sigma_{1}^{2}$ & $z_{t}^{(.9)}$ & $z_{t}^{(.7)}$ & $s_{t}$ & $s_{t}^{(1)}$ & $s_{t}^{(3)}$ & $s_{t}^{(5)}$ \\
\hline 100 & 10 & 4 & 3.4 & 36.3 & 4.6 & 60.6 & 99.8 & 100.0 \\
& & 10 & 0.2 & 36.2 & 4.4 & 60.2 & 99.8 & 100.0 \\
\cline { 2 - 8 } & 20 & 4 & 0.1 & 13.0 & 0.0 & 3.5 & 49.1 & 93.2 \\
& & 10 & 0.0 & 12.9 & 0.0 & 3.4 & 48.7 & 93.3 \\
\hline 250 & 10 & 4 & 3.3 & 36.0 & 4.3 & 61.1 & 99.8 & 100.0 \\
& & 10 & 0.2 & 35.8 & 4.3 & 60.9 & 99.8 & 100.0 \\
\cline { 3 - 8 } & 25 & 4 & 0.0 & 7.2 & 0.0 & 0.3 & 17.9 & 67.5 \\
& & 10 & 0.0 & 7.1 & 0.0 & 0.3 & 17.8 & 67.5 \\
\hline 500 & 25 & 4 & 0.0 & 7.0 & 0.0 & 0.5 & 17.4 & 67.1 \\
& & 10 & 0.0 & 6.9 & 0.0 & 0.5 & 17.4 & 67.1 \\
\cline { 2 - 8 } & 50 & 4 & 0.0 & 0.1 & 0.0 & 0.0 & 0.0 & 0.0 \\
& & 10 & 0.0 & 0.1 & 0.0 & 0.0 & 0.0 & 0.0 \\
\hline
\end{tabular}

Note: $M$ denotes the number of event dates, $\sigma_{1}^{2}$ the variance of the structural shock of interest on event dates, $z_{t}^{(.9)}$ a proxy with a theoretical correlation of 0.9 with the structural shock of interest on event dates, $z_{t}^{(.7)}$ a proxy with a theoretical correlation of 0.7 with the structural shock on event dates, $s_{t}$ the sign-proxy, and $s_{t}^{(m)}$ denotes a sign-proxy with $m$ incorrectly specified signs.

As discussed earlier, the strength of a proxy as an instrument depends on its correlation with the shock of interest. Therefore, it is not surprising that $z_{t}^{(.9)}$, which denotes the instrument having correlation 0.9 with the first shock, produces $F$-values less frequently below 10 than the corresponding instrument $z_{t}^{(.7)}$, which has only correlation 0.7 with the first shock. It is also not surprising that both proxies result in fewer $F$-values below 10 when they assume more nonzero values (i.e., the number of event dates, $M$, is greater),

\footnotetext{
${ }^{2}$ The robust $F$-statistic is the standard heteroskedasticity-robust statistic for testing the coefficient of $z_{t}$ to be zero in a regression of the first OLS estimated reduced-form error $\hat{u}_{1 t}$ on a constant and the instrument $z_{t}$. The statistic corresponds to the effective $F$-statistic of Montiel Olea and Pflueger (2013).
} 
when the sample size increases and when the variance $\sigma_{1}^{2}$ of the first shock in event periods is larger.

Interestingly, there are fewer $F$-values below 10 for the sign-proxy, $s_{t}$, than for the proxy $z_{t}^{(.7)}$ in all cases considered in Table 1 . Taking into account the generation mechanism of the proxy variable in equation (10), this outcome is not implausible because for $\operatorname{corr}\left(z_{t}, w_{1 t}\right)=0.7$, the sign of $z_{t}$ differs from that of $w_{1 t}$ in about $25 \%$ of the simulated values, whereas the sign of $s_{t}$ is always the same as that of $w_{1 t}$ (see equation (7)) and, hence, $s_{t}$ becomes a stronger instrument than $z_{t}^{(.7)}$. Moreover, $s_{t}$ has only slightly more $F$-values below 10 than the proxy $z_{t}^{(.9)}$. For $M=25$ event dates or more, or a sample size of $T=500$, both $z_{t}^{(.9)}$ and $s_{t}$ never yield $F$-values below 10 . In other words, in a usual proxy VAR analysis, the sign-proxy would be classified as a strong instrument more often than the proxy $z_{t}^{(.7)}$ and almost as often as the proxy $z_{t}^{(.9)}$, which is apparently based on more detailed information.

The situation changes if some of the signs are assigned incorrectly, as can be seen by looking at the frequencies reported for $s_{t}^{(1)}, s_{t}^{(3)}$, and $s_{t}^{(5)}$ in Table 1. For instance, for $T=250$, even the proxy $z_{t}^{(.7)}$ produces $F$-values below 10 in only about $36 \%$ of the replications for $M=10$ event periods, whereas a sign-proxy with 3 incorrect signs out of $10, s_{t}^{(3)}$, results in nearly all $F$-values below the threshold of 10 . On the other hand, when there are many event periods with nonzero proxies, the sign-proxy maintains its strong instrument status even with a few incorrect signs. For example, for $T=250$ and $M=25$ event dates, $s_{t}^{(3)}$ is only classified as a weak instrument by the $F$-test in about $18 \%$ of the replications of our simulations. Not surprisingly, the sign-proxy is not a useful instrument if half of the signs are incorrectly specified, as can be seen by looking at the frequencies associated with $s_{t}^{(5)}$ when there are only $M=10$ event periods. In that case, the robust $F$-statistic is always below 10.

In summary, using standard diagnostics, the sign-proxy may well be classified as a strong instrument as often as a conventional standard proxy even if the standard proxy has quite high correlation with the shock of interest. If the proxy is not strongly correlated with the shock, then the sign-proxy clearly dominates under this criterion. Even if some signs are misspecified, the $F$-test may classify the sign-proxy as a strong instrument more often than the standard proxy if there are sufficiently many event dates. Since the sign-proxy with correctly specified signs typically does not have a weak instrument problem, we do not consider weak instrument robust methods, as discussed by Montiel Olea, Stock and Watson (2020), for any of the estimators for better comparability. 


\subsubsection{RMSEs}

The absolute RMSEs for the two standard proxy estimators $\widehat{\mathbf{b}}_{12}^{P(.9)}$ and $\widehat{\mathbf{b}}_{12}^{P(.7)}$ are presented in Table 2, where it can be seen that the estimation precision declines substantially (the RMSEs increase), especially for sample sizes $T=$ 100 and 250 if $z_{t}^{(.7)}$ is used instead of $z_{t}^{(.9)}$.

Table 2: Absolute RMSEs of Proxy Estimators $\widehat{\mathbf{b}}_{12}^{P(.9)}$ and $\widehat{\mathbf{b}}_{12}^{P(.7)}$

\begin{tabular}{ccccc}
\hline \hline & & & \multicolumn{2}{c}{ absolute RMSEs } \\
\cline { 3 - 5 } Sample size $T$ & $M$ & $\sigma_{1}^{2}$ & $\widehat{\mathbf{b}}_{12}^{P(.9)}$ & $\widehat{\mathbf{b}}_{12}^{P(.7)}$ \\
\hline 100 & 10 & 4 & 0.253 & 2.468 \\
& & 10 & 0.153 & 1.561 \\
\cline { 2 - 5 } & 20 & 4 & 0.160 & 1.161 \\
& & 10 & 0.098 & 0.735 \\
\hline 250 & 10 & 4 & 0.281 & 1.472 \\
& & 10 & 0.156 & 0.931 \\
\cline { 2 - 5 } & 25 & 4 & 0.140 & 0.189 \\
& & 10 & 0.086 & 0.120 \\
\hline 500 & 25 & 4 & 0.139 & 0.190 \\
& & 10 & 0.086 & 0.120 \\
\cline { 2 - 5 } & 50 & 4 & 0.096 & 0.124 \\
& & 10 & 0.060 & 0.078 \\
\hline
\end{tabular}

Note: $M$ denotes the number of event dates and $\sigma_{1}^{2}$ is the variance of the structural shock of interest on event dates. The RMSE is calculated as $\sqrt{\frac{1}{N} \sum_{n=1}^{N}\left(\widehat{\mathbf{b}}_{12, n}^{P(.)}-\mathbf{b}_{12}\right)^{\prime}\left(\widehat{\mathbf{b}}_{12, n}^{P(.)}-\mathbf{b}_{12}\right)}$, where $N$ denotes the number of Monte Carlo simulations.

Relative RMSEs of the different estimators for the impact effects of the shocks are presented in Table 3 for a proxy having a correlation of 0.9 with the shock. Thus, the results in Table 3 refer to a highly correlated standard proxy, $z_{t}^{(.9)}$, which is rarely classified as a weak instrument by the $F$-tests reported in Table 1. Since the standard proxy uses the most detailed information, we expect it to provide potentially more precise estimates and, therefore, we report the other RMSEs relative to the corresponding RMSEs of the estimator $\widehat{\mathbf{b}}_{12}^{P(.9)}$ in Table 3 .

Indeed, almost all of the relative RMSEs presented in Table 3 are larger than 1, meaning that the RMSEs are larger than the corresponding ones of $\widehat{\mathbf{b}}_{12}^{P(.9)}$. However, the sign-proxy estimator $\widehat{\mathbf{b}}_{12}^{S P}$ has relative RMSEs very close to one such that the efficiency loss relative to the standard proxy VAR estimator is very limited. This holds for all simulation designs considered in 
Table 3: RMSEs of Estimators for Impact Effects of the First Shock Relative to the Corresponding RMSEs of $\widehat{\mathbf{b}}_{12}^{P(.9)}$

\begin{tabular}{cccrrrrrrr}
\hline \hline & & & \multicolumn{7}{c}{ relative RMSEs } \\
\cline { 3 - 9 }$T$ & $M$ & $\sigma_{1}^{2}$ & $\widehat{\mathbf{b}}_{12}^{S P}$ & $\widehat{\mathbf{b}}_{12}^{S P(1)}$ & $\widehat{\mathbf{b}}_{12}^{S P(3)}$ & $\widehat{\mathbf{b}}_{12}^{S P(5)}$ & $\widehat{\mathbf{b}}_{12}^{W}$ & $\widehat{\mathbf{b}}_{12}^{W P}$ & $\widehat{\mathbf{b}}_{12}^{W S P}$ \\
\hline 100 & 10 & 4 & 1.01 & 1.49 & 109.45 & 292.51 & 71.99 & 1.43 & 1.42 \\
& & 10 & 1.06 & 1.55 & 114.45 & 305.92 & 22.44 & 1.71 & 1.73 \\
\cline { 2 - 9 } & 20 & 4 & 1.08 & 1.22 & 1.63 & 4.34 & 30.41 & 1.47 & 1.41 \\
& & 10 & 1.11 & 1.25 & 1.67 & 4.46 & 76.10 & 1.65 & 1.49 \\
\hline 250 & 10 & 4 & 0.91 & 1.44 & 272.10 & 318.03 & 20.41 & 1.32 & 1.31 \\
& & 10 & 1.04 & 1.63 & 304.39 & 358.18 & 8.34 & 1.75 & 1.73 \\
\cline { 3 - 9 } & 25 & 4 & 1.09 & 1.20 & 1.49 & 1.99 & 33.23 & 1.59 & 1.11 \\
& 10 & 1.12 & 1.23 & 1.52 & 2.04 & 7.31 & 1.81 & 1.58 \\
\hline 500 & 25 & 4 & 1.10 & 1.20 & 1.49 & 2.00 & 50.50 & 1.89 & 1.80 \\
& & 10 & 1.12 & 1.23 & 1.52 & 2.05 & 325.24 & 2.35 & 1.49 \\
\cline { 2 - 9 } & 50 & 4 & 1.11 & 1.15 & 1.26 & 1.39 & 9.03 & 1.22 & 1.23 \\
& 10 & 1.13 & 1.18 & 1.29 & 1.42 & 5.76 & 1.16 & 1.13 \\
\hline
\end{tabular}

Note: $T$ signifies the sample size, $M$ denotes the number of event dates and $\sigma_{1}^{2}$ is the variance of the structural shock of interest on event dates.

Table 3. In fact, in one case, the relative RMSE of the sign-proxy estimator is even smaller than one. This situation arises for $T=250, \sigma_{1}^{2}=4$, and $M=10$. In other words, it happens for a case where the number of event periods is very small relative to the sample size and the standard proxy is occasionally classified as a weak instrument by the $F$-test in Table 1 .

Looking at the columns of $\widehat{\mathbf{b}}_{12}^{S P(1)}, \widehat{\mathbf{b}}_{12}^{S P(3)}$, and $\widehat{\mathbf{b}}_{12}^{S P(5)}$ in Table 3, it is apparent that, if some of the signs are assigned incorrectly, the efficiency of the sign-proxy estimator deteriorates, although the increase in relative RMSEs is rather moderate in some cases if only one sign is incorrect. However, note that, not surprisingly, the sign-proxy is no longer useful as an instrument if $50 \%$, or even $30 \%$, of the signs are incorrect (see the RMSEs for $\widehat{\mathbf{b}}_{12}^{S P(3)}$ and $\widehat{\mathbf{b}}_{12}^{S P(5)}$ when $\left.M=10\right)$. Additionally, the $F$-tests in Table 1 indicate that such proxies are not sufficiently strong instruments for proper inference.

Looking at the RMSEs of Wright's heteroskedasticity estimator $\widehat{\mathbf{b}}_{12}^{W}$, it is seen that they are much worse than those of the estimator based on the standard proxy. In some cases, the deterioration in estimation precision is rather extreme. For example, for a sample size of $T=500, M=25$ event periods, and a shock variance in the event periods of $\sigma_{1}^{2}=10$, the RMSE of the Wright heteroskedasticity estimator is more than 300 times the RMSE of the standard proxy. Generally, for all designs reported in Table 3, the Wright 
estimator is considerably less precise than the standard proxy estimator and it is also much less efficient than the sign-proxy estimator. In many cases, its RMSE is even larger than those of the corresponding sign-proxy estimators with some incorrectly assigned signs.

In our simulations, the Wright heteroskedasticity estimator does not uniformly improve with increasing variance $\sigma_{1}^{2}$ of the event dates. In some cases, a larger $\sigma_{1}^{2}$ results in a substantial reduction of the RMSEs of $\widehat{\mathbf{b}}_{12}^{W}$ (e.g., for $T=100$ and $M=10$ in Table 3 ), whereas in other cases the RMSEs of $\widehat{\mathbf{b}}_{12}^{W}$ increase (e.g., $T=100$ and $M=20$ in Table 3 ). The substantial RMSE increases may well be due to occasional poor estimates implied by difficulties in the numerical computation of the estimates. Recall that computing Wright's estimator involves a nonlinear function optimization.

The estimation precision improves substantially, if the moment conditions of Wright's heteroskedasticity estimator are combined with the moment conditions of the standard proxy or sign-proxy estimator (see the columns for $\widehat{\mathbf{b}}_{12}^{W P}$ and $\widehat{\mathbf{b}}_{12}^{W S P}$ in Table 3 ). However, the resulting RMSEs are clearly larger than one and, hence, these estimators are less precise than the standard proxy estimator or the sign-proxy estimator. This outcome may seem somewhat surprising, given that $\widehat{\mathbf{b}}_{12}^{W P}$ and $\widehat{\mathbf{b}}_{12}^{W S P}$ are GMM estimators based on more moment conditions than $\widehat{\mathbf{b}}_{12}^{P}$ and $\widehat{\mathbf{b}}_{12}^{S P}$. The reason that $\widehat{\mathbf{b}}_{12}^{W P}$ and $\widehat{\mathbf{b}}_{12}^{W S P}$ are still less precise estimators may be that the GMM objective function for these estimators is quite nonlinear and difficult to optimize. The optimization algorithm may not always find the global minimum. In any case, if the sign-proxy is available, there is nothing to be gained from also including the Wright heteroskedasticity moment conditions.

In summary, the results in Table 3 show that, in many scenarios, the sign-proxy estimator is almost as precise as the standard proxy estimator even if the standard proxy is highly correlated with the shock of interest. In contrast, the Wright heteroskedasticity estimator, which uses slightly less information than the sign-proxy estimator, is much less precise.

Looking at the results in Table 4 for a standard proxy with lower correlation of 0.7 with the shock of interest, it turns out that the sign-proxy estimator in this case is by far the most precise estimator. For almost all scenarios presented in Table $4, \widehat{\mathbf{b}}_{12}^{S P}$ has by far the smallest RMSEs. All its relative RMSEs are clearly smaller than one and, hence, they are substantially smaller than those of the standard proxy estimator based on $z_{t}^{(.7)}$. Even if one sign is specified incorrectly, all the relative RMSEs of the corresponding estimator $\widehat{b}_{12}^{S P(1)}$ are smaller than one and, hence, the estimator is more precise than $\widehat{\mathbf{b}}_{12}^{P(.7)}$. These results are quite plausible, given the finding in Table 1 that $s_{t}$ and even $s_{t}^{(1)}$ is classified as a strong instrument more often 
Table 4: RMSEs of Estimators for Impact Effects of the First Shock Relative to the Corresponding RMSEs of $\widehat{\mathbf{b}}_{12}^{P(.7)}$

\begin{tabular}{cccccccccc}
\hline \hline & & \multicolumn{7}{c}{ relative RMSEs } \\
\cline { 3 - 9 }$T$ & $M$ & $\sigma_{1}^{2}$ & $\widehat{\mathbf{b}}_{12}^{S P}$ & $\widehat{\mathbf{b}}_{12}^{S P(1)}$ & $\widehat{\mathbf{b}}_{12}^{S P(3)}$ & $\widehat{\mathbf{b}}_{12}^{S P(5)}$ & $\widehat{\mathbf{b}}_{12}^{W}$ & $\widehat{\mathbf{b}}_{12}^{W P}$ & $\widehat{\mathbf{b}}_{12}^{W S P}$ \\
\hline 100 & 10 & 4 & 0.16 & 0.24 & 18.25 & 49.32 & 12.80 & 0.23 & 0.23 \\
& & 10 & 0.16 & 0.24 & 18.25 & 49.32 & 3.20 & 0.27 & 0.27 \\
\cline { 2 - 9 } & 20 & 4 & 0.22 & 0.25 & 0.34 & 0.91 & 5.53 & 0.32 & 0.29 \\
& & 10 & 0.22 & 0.25 & 0.34 & 0.91 & 13.54 & 0.36 & 0.30 \\
\hline 250 & 10 & 4 & 0.18 & 0.28 & 48.54 & 62.74 & 4.28 & 0.27 & 0.26 \\
& & 10 & 0.18 & 0.28 & 48.54 & 62.74 & 1.44 & 0.31 & 0.30 \\
\cline { 2 - 9 } & 25 & 4 & 0.81 & 0.89 & 1.10 & 1.48 & 24.62 & 1.23 & 1.11 \\
& & 10 & 0.81 & 0.89 & 1.10 & 1.48 & 5.29 & 1.37 & 1.14 \\
\hline 500 & 25 & 4 & 0.81 & 0.88 & 1.09 & 1.48 & 37.25 & 1.43 & 1.32 \\
& & 10 & 0.81 & 0.88 & 1.09 & 1.48 & 233.13 & 1.75 & 1.49 \\
\cline { 2 - 9 } & 50 & 4 & 0.86 & 0.90 & 0.98 & 1.08 & 7.02 & 1.04 & 0.95 \\
& & 10 & 0.86 & 0.90 & 0.98 & 1.08 & 4.38 & 0.95 & 0.86 \\
\hline
\end{tabular}

Note: $T$ signifies the sample size, $M$ denotes the number of event dates and $\sigma_{1}^{2}$ is the variance of the structural shock of interest on event dates.

than $z_{t}^{(.7)}$ for a number of scenarios.

In contrast, the Wright heteroskedasticity estimator still has relative RMSEs greater than one for all scenarios. Thus, even a weaker instrument will typically result in better estimators than the Wright estimator. Again, combining Wright's moment conditions with those of the proxy or sign-proxy estimator improves the estimation precision considerably but does not result in more precise estimators than the sign-proxy estimator.

Overall, the results in Tables 3 and 4 show that the sign-proxy estimator is very competitive with the standard proxy estimator even though it uses less detailed information on the shock of interest. If the standard proxy is not very strong, the sign-proxy estimator provides much more precise estimates (in terms of RMSE) than the standard proxy estimator. If the standard proxy estimator is based on a strong instrument, the sign-proxy estimator is still almost as precise as the standard proxy estimator. Thus, despite its limited information requirement, it is an excellent choice for applied work. It is clearly preferable to Wright's heteroskedasticity estimator if the signs of the shock are known. 


\section{Monetary Policy at the Zero Lower Bound}

To illustrate the relevance of our results for empirical work, we consider the benchmark model of Wright (2012) who investigates the impact of US monetary policy on longer-term interest rates at times when the policy rate is at the zero lower bound. As mentioned in Section 3, Wright considers a VAR(1) model. The following six daily US interest rates are included: (1) the 2-year nominal Treasury zero-coupon yields, (2) 10-year nominal Treasury zerocoupon yields, (3) five-year Treasury Inflation Protected Securities (TIPS) break-even rates, (4) 5-10-year TIPS break-even rates, (5) Moody's index of AAA corporate bond yields, and (6) Moody's index of BAA corporate bond yields. The sample period runs from November 3, 2008, to September 30, 2011, giving a sample of size $T=730$.

As mentioned in Section 3, Wright also constructs a proxy variable for a monetary policy shock related to $M=28$ announcement days based on the first principal component of a set of bond futures traded at the Chicago Mercantile Exchange (see Wright's Table 1). We construct a sign-proxy with values \pm 1 on event dates using the signs of the Wright-proxy. All other elements are fixed at 0 . In his analysis, Wright also considers the possibility of using only 13 especially important event dates around new phases of quantitative easing. Since the number of event dates, $M$, was found to be an important determinant of the estimation precision in the simulations, we also consider a proxy and associated sign-proxy with only the 13 major event dates written in italics in Wright's Table 1.

We test the strength of both proxies and corresponding sign-proxies, presenting the robust $F$-values in Table 5 , where it can be seen that all proxies, apart from the sign-proxy for $M=28$, come with robust $F$-values well above the threshold of 10 . Thus, they would be classified as strong instruments in a standard proxy VAR analysis. The sign-proxy for $M=28$ yields a robust $F$-statistic of 9.55 , slightly missing the cut-off to be regarded as a strong instrument. We also present the empirical correlations between the proxies and the estimated first shocks on event dates in Table 5. Clearly, with -0.54 the correlation between the sign-proxy and the shock of interest is relatively small for $M=28$ event dates. All other proxies have stronger (negative) correlations with their respective shocks. This result indicates that with $M=28$, we may be in a situation where, based on our simulation results of Section 3, the corresponding Wright-proxy can be expected to yield more precise impulse response estimates than the sign-proxy. On the other hand, with $M=13$, where both the Wright-proxy and the sign-proxy are classified as strong instruments, the relative performance of the estimates $\widehat{\mathbf{b}}_{12}^{P}$ and $\widehat{\mathbf{b}}_{12}^{S P}$ is not clear a priori, although the correlation between the sign-proxy and the 
Table 5: Diagnostics for Proxy Strength

\begin{tabular}{lcclcc}
\hline \hline & \multicolumn{2}{c}{$M=28$ event dates } & & \multicolumn{2}{c}{$M=13$ event dates } \\
\cline { 2 - 3 } \cline { 5 - 6 } & Wright-proxy & sign-proxy & & Wright-proxy & sign-proxy \\
\hline Robust $F$-statistic & 49.90 & 9.55 & & 51.18 & 19.09 \\
Corr (proxy, shock) & -0.89 & -0.54 & & -0.90 & -0.74 \\
\hline
\end{tabular}

shock is weaker than the correlation between the Wright-proxy and the shock which might suggest a superior performance of the Wright-proxy.

We estimate impulse responses of the monetary policy shock and use the moving-block bootstrap (MBB) of Brüggemann, Jentsch and Trenkler (2016) and Jentsch and Lunsford (2019) to construct confidence intervals around the impulse responses estimated with the alternative estimators discussed in Section $2 .^{3}$

In Figure 1, we compare pointwise 90\% MBB confidence intervals associated with the Wright-proxy and the corresponding sign-proxy estimator for $M=13$. The shock is standardized such that it reduces the 10-year Treasury yields by 25 basis points on impact. In most cases, the two estimators yield very similar point estimates and confidence intervals. In some cases, the confidence intervals based on the Wright-proxy are slightly smaller than the corresponding intervals of the sign-proxy (e.g., the short-horizon responses of the 2-year Treasury rates) and, in other cases, the situation is reversed (e.g., the longer horizon responses of the 5-year break even rates). Overall, there is not much to choose between the Wright-proxy and the signproxy estimates. Thus, if there is any additional value from using the more sophisticated Wright-proxy over the simple sign-proxy, it is very limited.

The impulse responses are also qualitatively the same as in Wright (2012) (see, e.g., his Figure 1). The monetary policy shock does not have much of an effect on 5-year break-even rates and lowers BAA and AAA yields by less than 25 basis points. However, the confidence intervals in Wright's Figure 1 are partly considerably larger than in our Figure 1. A more systematic comparison of Wright's heteroskedasticity approach and the sign-proxy estimates is given below.

In Figure 2, we present the impulse response estimates obtained with

\footnotetext{
${ }^{3}$ Wright (2012) uses a slightly different bootstrap. We use the MBB because Jentsch and Lunsford (2019) show that it works under general conditions for inference for impulse responses in proxy VARs. Our block length is $\ell=25$, which corresponds roughly to the rule of thumb of $\ell=5.03 T^{1 / 4}$ of Jentsch and Lunsford (2019). The confidence intervals look very similar for a block length of 50 or using a residual wild bootstrap instead. The details of our MBB implementation are provided in the Appendix.
} 

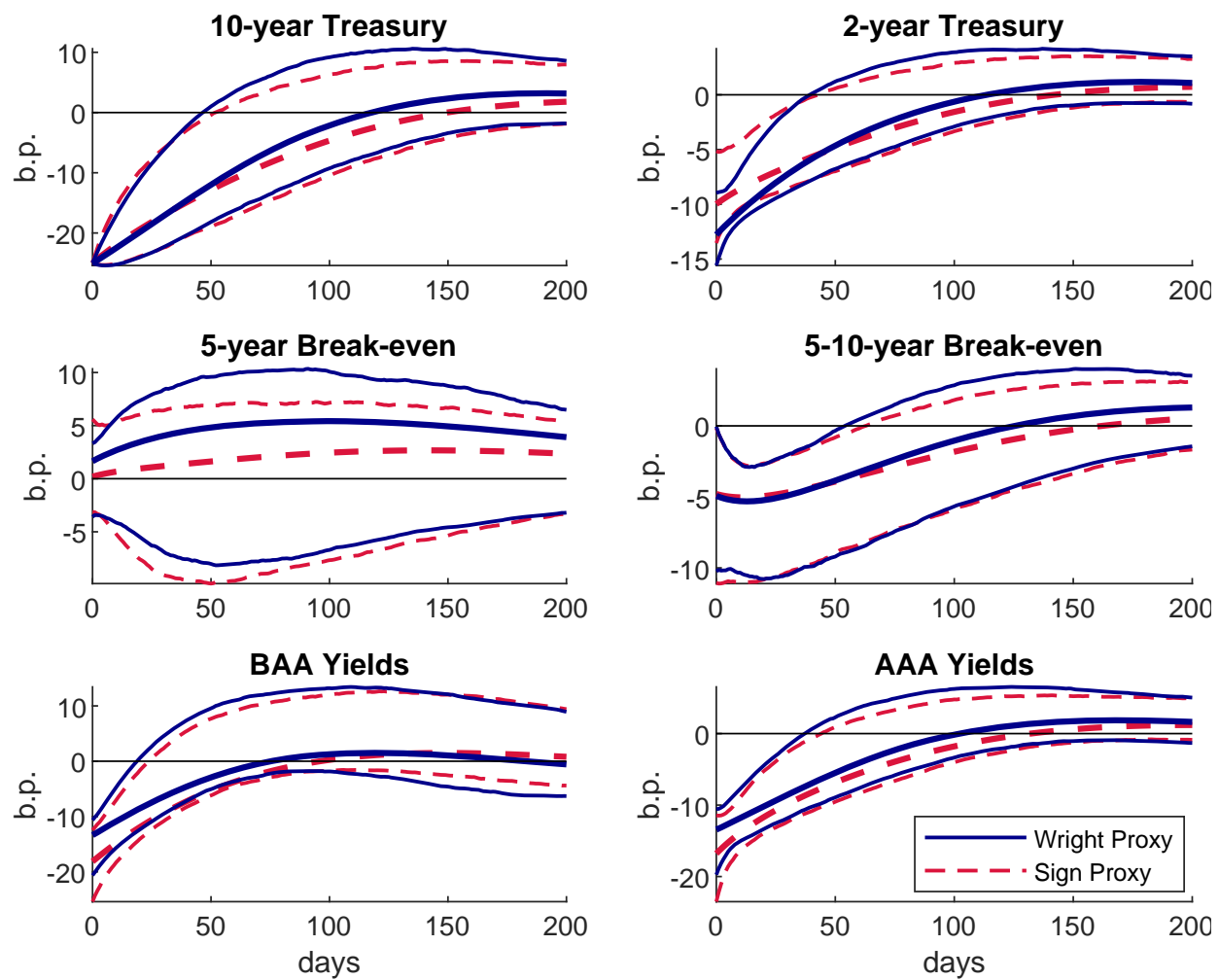

Figure 1: Wright-proxy and sign-proxy estimates of responses to 25 basis points monetary policy shock with $90 \%$ MBB confidence intervals for $M=13$ event dates.

proxies based on $M=28$ event dates. As expected on the basis of our simulation results in Section 3 and the $F$-statistics in Table 5, in this case the Wright-proxy estimator yields overall smaller confidence intervals than the sign-proxy estimator. However, in most cases, the conclusions regarding the responses of the variables are again qualitatively the same. The only exception is the initial response of the 5-10-year break-even rate, which is marginally significantly negative if the Wright-proxy estimator is considered while the sign-proxy confidence interval includes zero. Overall, the impulse response bands in Figure 2 reflect what we also find in the simulations in Section 3, namely that the decline in estimation precision can be rather limited even if a sign-proxy is used instead of a strong conventional proxy, which is constructed based on additional knowledge of the market structure.

In Figure 3, we show the impulse responses and $90 \%$ pointwise confidence intervals of the Wright heteroskedasticity estimator and the sign-proxy estimator based on $M=28$ event dates. For most variables, we qualitatively 

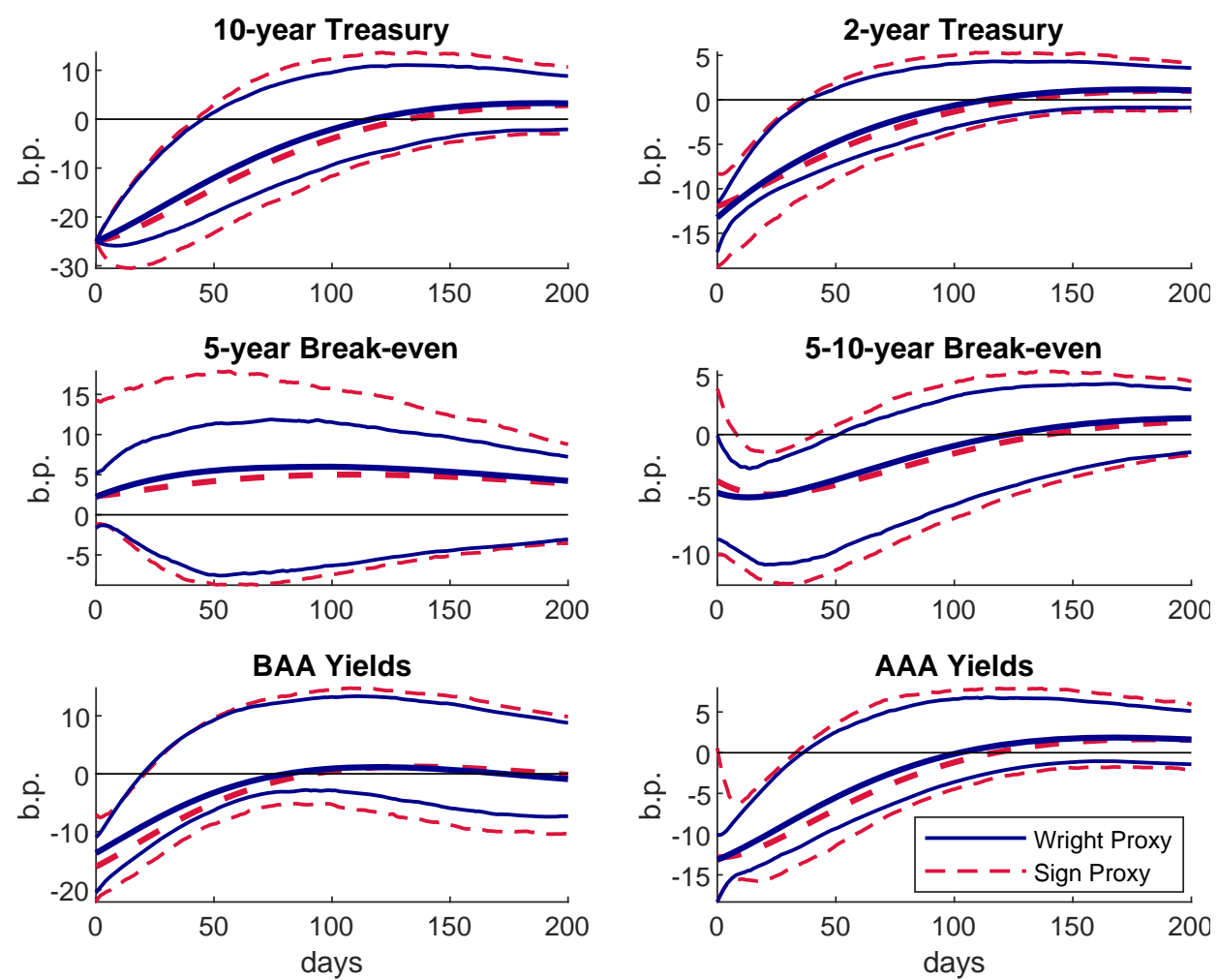

Figure 2: Wright-proxy and sign-proxy estimates of responses to 25 basis points monetary policy shock with $90 \%$ MBB confidence intervals for $M=28$ event dates.

obtain the same results from both estimators which are also in line with the results in Wright (2012). Note, however, that, e.g., for BAA and AAA yields the sign-proxy intervals for the initial response periods are considerably smaller than for Wright's heteroskedasticity estimator. For the 2-year Treasury yields, the sign-proxy also gives much smaller confidence intervals for the initial response periods than Wright's estimator, which is only marginally significant at the $10 \%$ level. Instead, the sign-proxy intervals lie clearly away from the zero line over the initial periods. Although both estimators yield intervals that include zero for the impact effects on the 5-10-year beak-even rate, the sign-proxy interval is again smaller than that of Wright's estimator. Thus, although the qualitative conclusions of Wright's study are confirmed, the precision of the inference can be improved by using the sign-proxy estimator.

In Figure 4, the MBB confidence intervals of the two GMM estimators $\widehat{\mathbf{b}}_{12}^{W P}$ and $\widehat{\mathbf{b}}_{12}^{W S P}$ which combine the moment conditions of Wright's proxy 

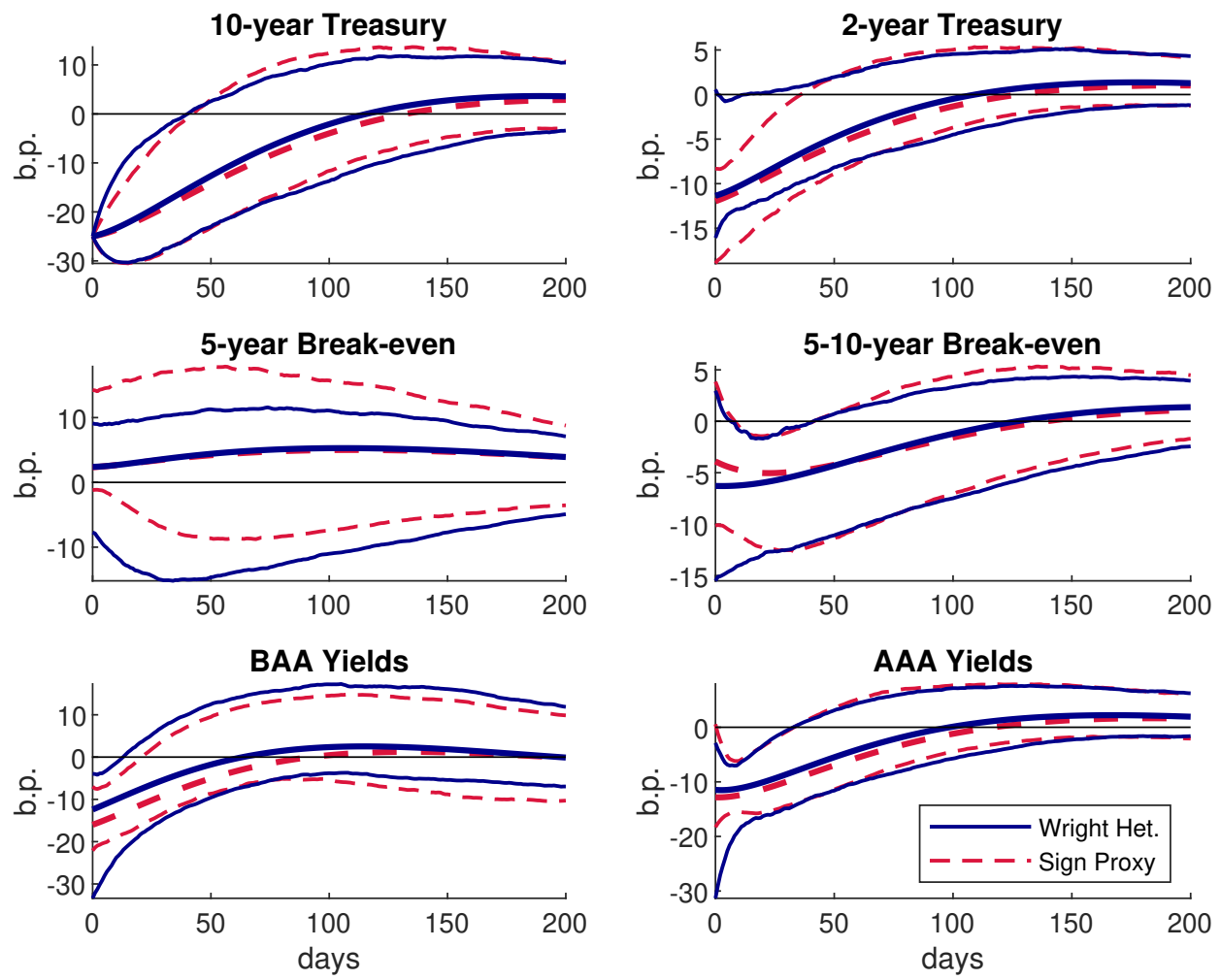

Figure 3: Sign-proxy and Wright heteroskedasticity estimates of responses to a 25 basis points monetary policy shock with $90 \%$ MBB confidence intervals for $M=28$ event dates.

and the sign-proxy, respectively, with the moment conditions of Wright's heteroskedasticity estimator are compared for $M=28$ event dates. To compare them to the Wright-proxy, we also show $90 \%$ confidence intervals for the Wright-proxy estimator in Figure 4. Across all estimators the point estimates of the impulse responses are very similar. For lucidity, we omit them in Figure 4 and focus on the $90 \%$ pointwise confidence intervals. The bootstrap confidence intervals of the two GMM combination estimators are practically identical and they are also quite similar to the confidence intervals associated with the Wright-proxy estimator. In line with our simulation results in Section 3, the intervals of the proxy estimator are slightly smaller in some cases. Hence, as in our Monte Carlo simulations, judged by the size of the confidence intervals, the GMM estimation precision of the sign-proxy and the conventional proxy are practically the same when they are used in combination with Wright's heteroskedasticity moment conditions. However, using the conventional proxy estimator without the additional moment conditions improves the precision slightly. 

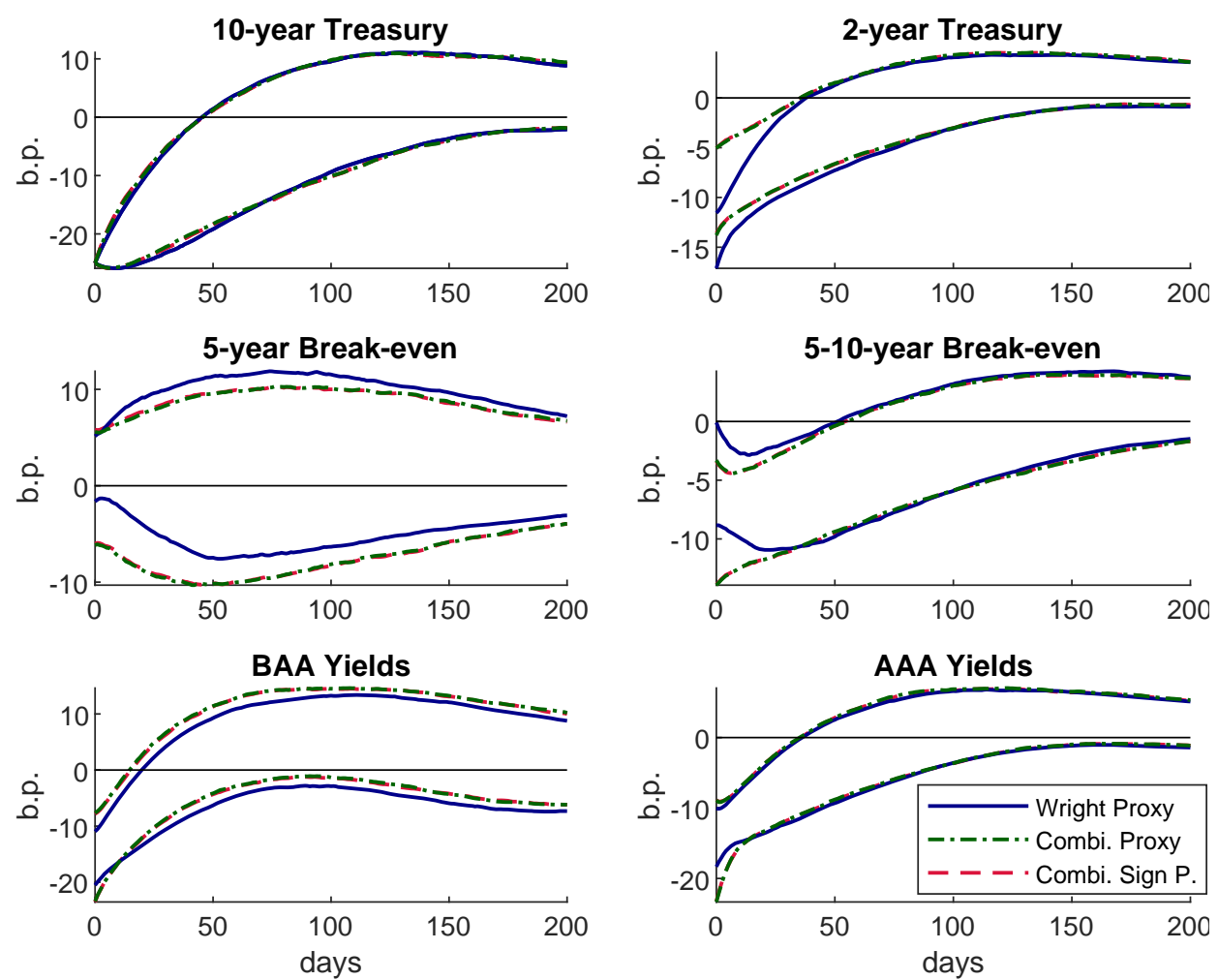

Figure 4: Pointwise 90\% MBB confidence intervals of Wright-proxy and GMM combination estimators of responses to a 25-basis-points monetary policy shock for $M=28$ event dates. (Point estimates are omitted for lucidity. They are all three very similar and well within the confidence intervals.)

Overall, the example reflects what we find in our Monte Carlo simulations in Section 3. The sign-proxy estimator has considerable merit. It tends to provide more precise inference than the Wright heteroskedasticity estimator. It can even be as precise as the conventional proxy estimator, which requires the construction of a suitable and possibly controversial strong proxy. As the simulations show, the sign-proxy may even dominate the conventional proxy estimator if the proxy is a weak instrument. Thus, the sign-proxy estimator is an attractive choice for empirical structural VAR analyses.

\section{Conclusions}

This study contributes to the rapidly growing literature on proxy VAR models by proposing a proxy variable that is often easy to construct. The construction of a suitable instrument for estimating the initial responses of a 
shock of interest is crucial in a proxy VAR analysis. The precision of the estimates depends on the quality of the proxy, which has to be well correlated with the structural shock and uncorrelated with all other shocks. Such a proxy variable may be difficult to find in practice. Therefore, we propose to construct a proxy that assigns \pm 1 for periods where special events occur that are associated with a shock. A +1 is assigned for positive shocks and a -1 is assigned for negative shocks. Thus, to construct the proxy, it is enough to know the timing and direction of the induced shock.

Through simulation, we demonstrate that such a sign-proxy may produce estimates of the impact effects of the shock that may be almost as efficient as the estimator based on a more sophisticated strong proxy that can only be constructed with substantially more information on the implications of the special events. It turns out that the sign-proxy can provide a reasonably precise estimator even if occasional errors are made in assigning the sign of the shock. Moreover, we point out that the moment conditions implied by the proxy variable can be supplemented by moment conditions from possible volatility changes due to the special events. The combined moment conditions can be used for GMM estimation of the impact effects of the shocks. While this GMM estimator does not improve the estimation precision in some of our simulations, it may have merit if the resulting GMM objective function can be minimized with sufficient reliability. In any case, the sign-proxy or combination estimator can be considerably more precise than an estimator that is based only on the volatility changes induced by the special events.

We illustrate the benefits of using the sign-proxy by investigating the impact of US monetary policy shocks on longer-term interest rates in times of a zero policy interest rate. It is shown that bootstrap confidence intervals of the impulse responses based on the sign-proxy estimator or the combination estimator are, in many cases, clearly smaller than competitors based only on the volatility changes. The sign-proxy can even yield more precise estimates than a conventional proxy estimator. Thus, the sign-proxy estimator is an attractive choice in empirical structural VAR analyses because it requires only very limited information external to the VAR model of interest.

\section{Appendix. Implementation of the Moving- Block Bootstrap}

Bootstrap samples are generated by a moving-block bootstrap (MBB), as inJentsch and Lunsford (2019). In the following, $\hat{u}_{1}, \ldots, \hat{u}_{T}$ are the estimated reduced-form VAR OLS residuals and $z_{1}, \ldots, z_{T}$ are the observed proxy val- 
ues.

Let $\ell<T$ be the block length for the MBB. Then $s=[T / \ell]$ is the number of blocks required for constructing a bootstrap sample of $y_{t}$. Here [.] denotes the smallest number greater than or equal to the argument such that $\ell s \geq T$. Blocks of length $\ell$ of residuals are arranged as follows:

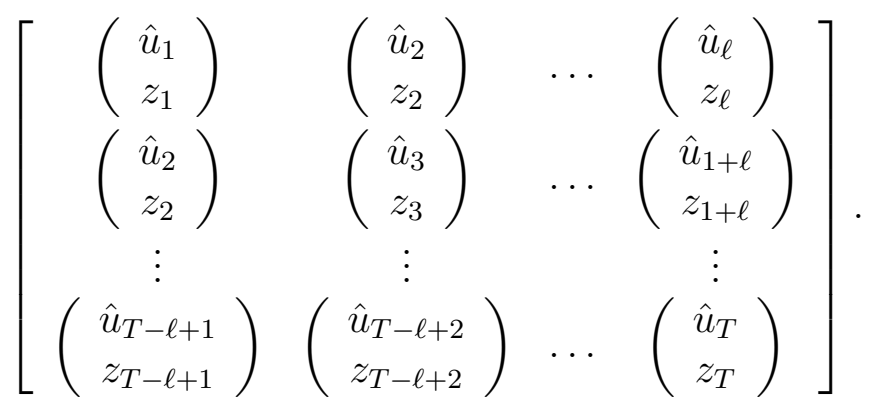

Then $s$ of the rows of the matrix are drawn with replacement and these draws are joined end-to-end and the first $T$ residuals and proxies are retained,

$$
\left(\begin{array}{l}
u_{t}^{*} \\
z_{t}^{M B B}
\end{array}\right), \quad t=1, \ldots, T \text {. }
$$

The $u_{t}^{*}$ are recentered as

$$
u_{j \ell+i}^{M B B}=u_{j \ell+i}^{*}-\frac{1}{T-\ell+1} \sum_{r=0}^{T-\ell} \hat{u}_{i+r}
$$

for $i=1,2, \ldots, \ell$ and $j=0,1, \ldots, s-1$ and the bootstrap residuals and proxies are obtained as

$$
\left(\begin{array}{c}
u_{t}^{M B B} \\
z_{t}^{M B B}
\end{array}\right), \quad t=1, \ldots, T \text {. }
$$

The bootstrap sample is then sequentially generated as $y_{t}^{M B B}=\hat{\nu}+\hat{A}_{1} y_{t-1}^{M B B}+$ $\cdots+\hat{A}_{p} y_{t-p}^{M B B}+u_{t}^{M B B}, t=1, \ldots, T$, starting from $p$ randomly chosen consecutive sample values, $y_{-p+1}^{M B B}, \ldots, y_{0}^{M B B}$.

When $N$ bootstrap samples $y_{-p+1}^{(n)}, \ldots, y_{0}^{(n)}, y_{1}^{(n)}, \ldots, y_{T}^{(n)}$ and $z_{1}^{(n)} \ldots, z_{T}^{(n)}$, $n=1, \ldots, N$, are available, then the following steps are used for each sample $n$ to obtain bootstrap impulse response estimates:

1. A $\operatorname{VAR}(p)$ model is fitted to the sample, giving bootstrap estimates $\hat{A}^{(n)}$,

$$
\hat{\Phi}_{i}^{(n)}=\sum_{j=1}^{i} \hat{\Phi}_{i-j}^{(n)} \hat{A}_{j}^{(n)}, \quad i=1, \ldots, H,
$$

and residuals $\hat{u}_{t}^{(n)}$. 
2. Bootstrap estimates $\widehat{\mathbf{b}}_{1}^{(n)}$ are computed with all the alternative estimation methods using $\mathcal{T}_{1}=\left\{t \mid z_{t} \neq 0\right\}$ for the Wright heteroskedasticity estimator and associated combination estimators.

3. Bootstrap estimates of the impulse responses of interest are computed as

$$
\widehat{\Theta}(H)^{(n)}=\left[\widehat{\mathbf{b}}_{1}^{(n)}, \hat{\Phi}_{1}^{(n)} \widehat{\mathbf{b}}_{1}^{(n)}, \ldots, \hat{\Phi}_{H}^{(n)} \widehat{\mathbf{b}}_{1}^{(n)}\right]
$$

and stored.

The $N$ bootstrap estimates $\widehat{\Theta}(H)^{(1)}, \ldots, \widehat{\Theta}(H)^{(N)}$ are used to construct pointwise confidence intervals from the relevant quantiles of the bootstrap distributions of the individual elements.

\section{References}

Brüggemann, R., Jentsch, C. and Trenkler, C. (2016). Inference in VARs with conditional heteroskedasticity of unknown form, Journal of Econometrics 191: 69-85.

Caldara, D. and Herbst, E. (2019). Monetary policy, real activity, and credit spreads: Evidence from bayesian proxy svars, American Economic Journal: Macroeconomics 11: 157-192.

Cesa-Bianchi, A., Thwaites, G. and Vicondoa, A. (2020). Monetary policy transmission in the United Kingdom: A high frequency identification approach, European Economic Review 123(103375).

Gertler, M. and Karadi, P. (2015). Monetary policy surprises, credit costs, and economic activity, American Economic Journal: Macroeconomics 7: $44-76$.

Jentsch, C. and Lunsford, K. G. (2019). The dynamic effects of personal and corporate income tax changes in the United States: Comment, American Economic Review 109: 2655-2678.

Kilian, L. and Lütkepohl, H. (2017). Structural Vector Autoregressive Analysis, Cambridge University Press, Cambridge.

Lütkepohl, H. (2005). New Introduction to Multiple Time Series Analysis, Springer-Verlag, Berlin. 
Lütkepohl, H. and Schlaak, T. (2020). Heteroskedastic proxy vector autoregressions, Discussion Paper 1876, DIW Berlin, Berlin.

Mertens, K. and Ravn, M. O. (2013). The dynamic effects of personal and corporate income tax changes in the United States, American Economic Review 103: 1212-1247.

Montiel Olea, J., L. and Pflueger, C. (2013). A Robust Test for Weak Instruments, Journal of Business \& Economic Statistics 31(3): 358-369.

Montiel Olea, J. L., Stock, J. H. and Watson, M. W. (2020). Inference in structural vector autoregressions identified with an external instrument, Technical report, Columbia University.

Mood, A. M., Graybill, F. A. and Boes, D. C. (1974). Introduction to the Theory of Statistics, Vol. Auckland, 3rd edn, McGraw-Hill.

Piffer, M. and Podstawski, M. (2018). Identifying uncertainty shocks using the price of gold, The Economic Journal 128(1549): 3266-3284.

Stock, J. H. and Watson, M. W. (2012). Disentangling the channels of the 2007-09 recession, Brookings Papers on Economic Activity pp. 81-135.

Stock, J. H., Wright, J. H. and Yogo, M. (2002). A survey of weak instruments and weak identification in generalized method of moments, Journal of Business 86 Economic Statistics 20(4): 518-529.

Wright, J. H. (2012). What does monetary policy do to long-term interest rates at the zero lower bound?, Economic Journal 122: F447-F466. 